\title{
Higher participation in physical activity is associated with less use of inpatient mental health services: A cross-sectional study.
}

\author{
Joseph Korge* \\ Dr David Nunan
}

Nuffield Department of Primary Care Health Sciences, University of Oxford, UK. 


\begin{abstract}
$\underline{\text { Abstract }}$
There is a stark disparity in the physical health of people with mental illness compared to those without mental illness, resulting in shorter life expectancy and increased rates of preventable deaths. Physical activity has previously been shown to have a positive impact on various markers of mental health and has been linked with a reduction in hospital admissions for those with chronic physical illness. The purpose of this study was to investigate whether there is a relationship between physical activity and the number of days spent admitted to acute inpatient mental health wards in people with enduring mental illness. Eighty participants from four acute mental health wards in Oxfordshire, UK were included. Subjective and objective measurements of physical activity levels were collected alongside the amount of days spent admitted on acute mental health wards over the year previous. Participants who recorded higher levels of physical activity, both subjectively and objectively, were found to have spent less time admitted to acute mental health services. With a significant negative correlation found, future research should aim to investigate any causative link between physical activity and mental health admission.
\end{abstract}

Key words: Exercise, physical health, admission, depression, schizophrenia, bipolar.

\title{
1. Introduction
}

Mental illness affects one in four people in the UK (1) and a third of the 100,000 preventable deaths in the UK each year also have a mental illness (2). Common causes of early death in people with mental illness are the same as the common causes of death in the wider population - predominantly physical illnesses related to lifestyle factors such as diabetes, heart disease and respiratory disease. Being mentally unwell is associated with a 3 fold increased risk of diabetes (3) and hypertension (2), a 10-fold increase in deaths from respiratory disease (2) and over 4 times the risk of premature death compared to those without mental illness (2). This increased risk of physical morbidity may be attributable to increased sedentary behaviours in people with mental illness $(3,4)$ which has been shown to increase the risk of metabolic disorders in this patient group (5).

The risk and severity of these lifestyle associated physical conditions have been shown to reduce with the inclusion of healthy lifestyle interventions, with physical activity at the heart of those interventions $(6,7,8)$. To reflect this, the 2014 update on psychosis and schizophrenia quality standards from the National Institute for Clinical Excellence (NICE) puts particular emphasis on physical health promotion in this patient group (9).

There has been an emergence of literature on the effects of physical activity and healthy lifestyle interventions in people with mental illness over the last decade. Links have been found between higher self-reported physical activity levels and various outcomes such as depression scales, neurobiological changes, quality of life and psychotic symptoms $(10,11,12$, $13,14,15,16,17,18,19,20,21,22,23,24,25,26,27)$. One gap in the existing evidence base is that the relationship between physical activity and mental health has been based predominantly on narrow, rather than broad, outcome measures. This was highlighted by Douzanis et al. (2012) who recommend the use of broad outcome measures such as length of admission to address this knowledge gap (14). To our knowledge, no previous studies have assessed the relationship between physical activity and admission to inpatient mental health wards .

The purpose of this study was to therefore investigate the association between physical activity participation and days of admission, over a year period, to acute mental health wards. 
Our hypothesis was that there would be a negative correlation between physical activity levels and mental health admission.

\section{$\underline{\text { 2. Methods }}$}

We conducted a cross-sectional study in which individual participants' days spent admitted to acute wards was measured alongside their self-reported physical activity and their actual participation in physical activity sessions as inpatients. The protocol was approved by the North-West Greater Manchester South Research Ethics Committee (16/NW/0300). Each participating centre gave NHS Research and Development approval. All participants provided written informed consent before taking part in the study.

\section{$\underline{2.1 \text { Sample Size }}$}

Eighty participants who met the inclusion criteria, and provided informed consent to be included in the study, were recruited from four acute inpatient wards across two mental health centres within a trust in Oxford, UK. Only two people, who met the inclusion criteria, declined to be included in the study for unknown reasons. The sample size was decided upon due to the time constraints of the study, which formed the dissertation thesis for a master's degree. A lack of previous research in the field of physical activity and admission rates in people with enduring mental illness prevented a sample size calculation.

\subsection{Inclusion Criteria}

- Participants willing and able to give informed consent for participation in the study.

- Patients admitted onto acute mental health wards with a mental health diagnosis who are judged settled enough by their care team to have been granted leave from the ward environment.

\section{$\underline{2.3 \text { Exclusion Criteria }}$}

- Patients deemed to lack capacity to provide informed consent.

- Patients without a diagnosed mental illness.

- Patients with serious long term physical disability as they may present increased confounding factors to the study.

- Any patients without an understanding of written or spoken English that is sufficient to understand verbal questioning.

\subsection{Data Collection}

Self-report physical activity data was collected in single visits using a validated questionnaire: The World Health Organisation Global Physical Activity Questionnaire (GPAQ) version 2 (28). The GPAQ gathers information about physical activity at work, during regular travel and from recreational exercise, in a typical week and was preferred to other physical activity questionnaires that determine physical activity levels over the preceding week. For many patients in acute mental health care, the preceding week is likely to have been a turbulent and unusual one. Data on usual physical activity levels in this 
population is more likely to be prone to bias if determined from the week preceding inpatient admission or stay. The GPAQ provides answers that better reflect long-term physical activity habits hence it's preferred use in this study.

The GPAQ was administered verbally to consenting participants, as per WHO GPAQ version 2 guidance (28). The GPAQ answers were then used to calculate each participants' weekly physical activity levels in Metabolic Equivalent to Task (MET) minutes. MET minutes represent the effort required for different exercise intensities. For low intensity exercise, such as walking to work, the minutes spent doing the exercise are multiplied by 4 to provide the MET minutes. For higher intensity exercise, such as running, the time is multiplied by 8 . For example, 30 minutes spent running would amount to 240 MET minutes $(30$ x 8).

To provide a reference point for participants' engagement with physical activity, the average weekly attendance at supervised exercise sessions as part of their usual inpatient care provision was also recorded. These sessions are available to all inpatients and are supervised by physiotherapy staff. They include one ward based exercise group and two gym sessions per week providing a total of three exercise opportunities per week. This information was compared with self-reported physical activity to cross validate GPAQ questionnaire answers. The amount of days that participants had spent as an inpatient over the previous year was obtained from electronic medical records after collection of self-reported and inpatient physical activity.

Our primary analysis was to assess any association between self-reported physical activity and the number of days spent as an inpatient over the previous 12 months. We assessed selfreported physical activity as an overall exposure and categorised participants' activity levels based on the WHO recommendation of 600 MET minutes per week. We additionally assessed whether attendance to inpatient exercise sessions was associated with inpatient stay, and separately, with self-reported physical activity levels.

\section{$\underline{2.5 \text { Data Analysis }}$}

Using IBM SPSS version 23, Pearson correlation coefficients were calculated to demonstrate any linear relationship between the various types of physical activity recorded on the GPAQ, and inpatient physical activity participation, and admission (days) over the year previous. Linear regression analysis was carried out to further demonstrate any association between the various measures of physical activity collected and admission, in days, over the year previous.

\section{Results}

Eighty participants were recruited between May 2016 and August 2016. A total of 18 (22.5\%) participants were diagnosed with schizophrenia, 19 (23.8\%) bipolar disorder, 18 $(22.5 \%)$ schizoaffective disorder, 13 (16.3\%) bipolar-affective disorder and $12(15 \%)$ with severe depression. Initial analysis showed no difference in mean admission or activity levels between participants with different diagnoses.

\section{$\underline{\text { 3.1 Self-reported activity vs. Admission }}$}


Admission (days) was negatively correlated with self-reported physical activity $(\mathrm{r}=-.365$, SE: .03, 95\%CI: $-.31--.45, p=<.05)$. The GPAQ separates weekly physical activity into three categories, all of which negatively correlated with admission: Self-reported work related exercise $(\mathrm{r}=-.269$, SE: $.04,95 \% \mathrm{CI}:-.19--.37 p=<.05)$, self-reported travel related exercise $(\mathrm{r}=-.311$, SE: $.05,95 \% \mathrm{CI}:-.20--.48, p=<.05)$ and self-reported recreational exercise ( $\mathrm{r}=-.316$, SE: $.06,95 \% \mathrm{CI}:-.19--.43, p=<.05)$. A simple linear regression was calculated, using the least squares method, to further quantify the association and a significant regression equation was found $(\mathrm{F}(1,78)=12.008, p<.001)$, with an adjusted $R$ square of .122.

Participants who exceeded the WHO recommended 600 MET minutes of exercise weekly had lower mean admission days than those who didn't. People who reported above 600 total MET minutes spent an average of 55 days admitted to hospital compared to those who reported less than 600 total MET minutes who spent an average of 122 days in hospital.

\subsection{MET Minutes vs Inpatient Activity}

Total MET minutes per week correlated positively with activity participation during inpatient admission $(\mathrm{r}=.724,95 \% \mathrm{CI}: .55-.83, p=<.001)$, indicating that people who say they are more active generally, are more active as inpatients.

\section{$\underline{3.3 \text { Inpatient Physical Activity vs. Admission }}$}

The variable with the strongest negative correlation to inpatient admission was inpatient physical activity, measured objectively using average weekly participation in activity sessions during admission. The correlation coefficient for inpatient activity and inpatient admission was moderate to high $(\mathrm{r}=-.764,95 \% \mathrm{CI}$ : $-.65--.84, p=<.001)$. A simple linear regression was calculated, using the least squares method, to predict admission (days/year) based on mean inpatient physical activity sessions per week. A significant regression equation was found $(\mathrm{F}(1,78)=35.199, p<.001)$, with an adjusted $R$ square of .302 . Mean admission (days) for patients participating in no sessions, one session, two sessions and three sessions of physical activity per week were 127, 83, 32 and 15 respectively.

\section{Discussion}

The primary objective of the study was to identify whether there is an association between physical activity and admission to mental health inpatient care. Although a causal relationship has not been found, the results show that there is a correlation between physical activity participation and days of admission per year. The results suggest that, the more active a person is, the less time they spend in acute mental health care over a year period. The relationship between inpatient activity participation and admission demonstrates a strong correlation, with a weaker correlation seen between admission and self-reported activity levels. If we are to assume admission is an indicator for mental health, that the more unwell a person is the more time they spend admitted to hospital, the results suggest that people who suffer with mental illness and exercise more, have better mental health requiring less time in hospital. 
Though no previous research investigating the relationship between activity and admission in mental health can be found, these findings do mirror the findings from previous research with alternative outcome measures $(5,10,11,12,13,14,15,16)$. For example, Vancampfort et al. (2013) found that MET minutes per week explained $10 \%$ of the variance in muscular fitness among people with schizophrenia (5), a systematic review by Holley et al. in 2011 concluded that exercise appears to benefit the psychological well-being of people with schizophrenia (10) and Thompson et al. (2015) report promising evidence for the use of exercise in the management of depressive episodes in people with bipolar disorder (15). The current study adds to the body of work by introducing admission as a broad outcome measure, as recommended in previous research $(11,16)$ and ensures clinical applicability of the findings by investigating a range of mental health diagnoses together, the way they are treated in practice.

Reported activity levels derived using the GPAQ did correlate strongly with objectively recorded physical activity during inpatient admission. These findings mirror previous studies on the GPAQ and validate the subjective information used in this study (29). Though the findings do strengthen the justification for use of the GPAQ within future physical activity research in mental health, future observational studies would benefit from an objective measure of activity to validate any subjective findings.

A relationship between physical activity uptake and time spent admitted to acute mental health wards has been identified. From this relationship, a few hypotheses can be made. Firstly we hypothesise that mental health service-users, who exercise more during acute admission, recover quicker and are therefore discharged sooner into their stay. We also hypothesise that mental health service users who are more active in their lives outside of hospital require shorter inpatient stays when they are admitted. It is also hypothesised that, mental health service users who are physically inactive in the community, continue to be so during acute psychiatric admission and require targeted exercise interventions to promote activity during those admissions. Finally, it is hypothesised that a healthy lifestyle which includes adequate physical activity, healthy dietary habits and reduced sedentary behaviour, reduces the rate and severity of relapse in people suffering with mental illness and in turn reduces the need for, and length of, inpatient psychiatric admission.

\section{$\underline{4.1 \text { Recommendations }}$}

For researchers in the field of acute mental healthcare, it is important to build on these findings. The ideal next study would attempt to confirm and quantify the association between physical activity and admission to acute mental health wards. Following a sample over a period of time would shed further light on the strength of the relationship between activity and admission. It would also allow for more in depth data collection to identify any likely confounding factors, such as psychotropic medication use and dietary habits. Should a strong correlation be confirmed by a long term prospective cohort study, causation between physical activity and admission should be investigated by means of a randomised trial controlling for any possible confounding factors. The eventual aim should be to ascertain whether or not increasing a person's physical activity levels reduces their need for acute mental health inpatient care.

\section{$\underline{4.2 \text { Limitations }}$}

The primary limitation of this study is the inability to attribute causality to the relationships identified. Without controlling for confounding factors, this simply isn't possible. The data 
itself was collected at a single point in time and, although it does provide an insight into the use of inpatient services, among patients with varying levels of physical activity participation, it opens the results up to inevitable discrepancies. In so much as, a person's activity participation may fluctuate over the period of a year and the recorded activity levels at the point of data collection may differ from the activity levels over the whole year previous in which admission data was collected. The use of a broad outcome measure is itself open to confounding. The assumption made by this study, is that admission is directly related to the mental health status of service users. It was beyond the scope of this study to investigate any confounding effect of extraneous variables on admission length and quantity. The need for informed consent also creates a limitation, in that patients who were unable to provide informed consent were excluded. Such patients are likely to be the most unwell and leaving them out of the study could have biased the results. Although some effort was made to validate subjective findings with an objective measure of physical activity propensity, all-but one of the independent variables collected for the study were subjective which presents a risk of response bias, more specifically social desirability bias (30).

\section{$\underline{4.3 \text { Conclusion }}$}

The results of this study show that people with mental illness who are less active, are more likely spend more time admitted to acute mental health wards. By identifying a relationship between mental health admission and a person's physical activity levels, and along-side the growing evidence base for the benefits of increased exercise in this patient group, mental health clinicians should confidently promote physical activity among service users. Although the total factors that make up a person's exercise habits stretches far beyond the scope of this study alone, it gives an indication of the patterns observed within this patient group and lays the foundation for further research into the causal effects between admission and physical activity.

The findings of this study should be taken into context and readers should be aware that a correlation, rather than causation, has been identified. That said, the aims of the study have been achieved and the primary question answered. There is an association between physical activity participation and the amount of time a person spends in acute inpatient mental health care. This negative relationship is particularly strong regarding physical activity participation during acute psychiatric hospital admission. 


\section{$\underline{\text { References }}$}

1. The Health \& Social Care Information Centre, 2009, Adult psychiatric morbidity in England, Results of a household survey.

2. Faculty of Public Health, 2008. Mental health and smoking: a position statement (online).

3. Vancampfort, D., Firth, J., Schuch, F., Rosenbaum, S., De Hert, M., Mugisha, J., Probst, M., Stubbs, B. Physical activity and sedentary behaviour in people with bipolar disorder: A systematic review and meta-analysis. J. Affect. Disord., 201, pp. 145-152 (2016).

4. Stubbs, B., Williams, J., Gaughran, F., Craig, T. How sedentary are people with psychosis? A systematic review and meta-analysis. Schizophr. Res. (2016).

5. Vancampfort, D., Probst, M., Knapen, J., Carraro, A., De Hert, M. Associations between sedentary behaviour and metabolic parameters in patients with schizophrenia. Psychiatry Res., 200, pp.73-78 (2012).

6. Lindstrom, J., Peltonen, M., Ericsson, J.G., Ilanne-Parikka, P., Aunola, S., Keinaanen-

Kiukaanniemi, S., Uusitupa, M. \& Tuomilehto, J. Improved lifestyle and decreased diabetes risk over 13 years: Long term follow-up of the randomised Finnish Diabetes Prevention Study. Diabetologia 56, pp 284-93 (2013).

7. European Society of Cardiology. European Guidelines on cardiovascular disease prevention in clinical practice (2012).

8. Romain, A., Pauwells, A., Buist, S., Peter, M., Calverly, A., Jenkins, C.R. \& Hurd, S.S. Global Strategy for the Diagnosis, Management, and Prevention of Chronic Obstructive Pulmonary Disease. American Journal of Respiratory and Critical Care Medicine 163, pp. 1256-76 (2001).

9. NICE quality standard (QS80). Psychosis and schizophrenia quality standards (2015).

10. Holley, J., Crone, D., Tyson, P., Lovell, G. The effects of physical activity on psychological well-being for those with schizophrenia: A systematic review. Br J Clin Psychol. 50, pp 84-105 (2011).

11. Gorcynski, P., Faulkner, G. Exercise therapy for schizophrenia. Cochrane Database Systematic Review (5). CD004412 (2010).

12. Schuch, F.B., Vancampfort, D., Richards, J., Rosenbaum, S., Ward, P.B., Stubbs, B. Exercise as a treatment for depression: a meta-analysis adjusting for publication bias. J. Psych. Res. 77; pp. 42-51 (2016).

13. Kelly, C., McCreadie, R. G. Smoking habits, current symptoms, and premorbid characteristics of schizophrenic patients in Nithsdale, Scotland. American Journal of Psychiatry, 156, 17511757 (1999).

14. Vancampfort, D., Correll, C.U, Probst, M., Sienaert, P., Wykaert, S., De Herdt, A., Knapen, J., De Wachter, K., De Hert, M. A review of physical activity correlates in patients with bipolar disorder. J. Affec. Dis. 145:3, pp. 285-91 (2013).

15. Thomson, D., Turner, A., Lauder, S., Gigler, M. E., Berk, L., Singh, A. B., ... Sylvia, L. A brief review of exercise, bipolar disorder, and mechanistic pathways. Frontiers in Psychology. 6, p.147 (2015).

16. Douzenis, A., Seretis, D., Nika, S., Nicolaidou, P., Papadopolou, A., Rizos, E.N. Factors affecting hospital stay in psychiatric patients: the role of active co-morbidity. BMC Health Serv Res. 12:166, pp. 1472-6963 (2012).

17. Crump, C., Winkleby, M.A., Sundquist, K., Sungquist, J. Comorbidities and mortality in persons with schizophrenia: A Swedish national cohort study. Am J Psych. 170, pp. 324-33 (2013). 
18. Soundy, A., Stubbs, B., Probst, M., Hemmings, L., Vancampfort, D. Barriers and Facilitators of Physical Activity Among Persons with Schizophrenia: A Survey of Physical Therapists. J. Am Psych Assoc. 65:5, pp. 693-6 (2014).

19. Vancampfort, D., Rosembaum, S., Probst, M., Connaughton, J., du Plessis, C., Yamamoto, T., Stubbs, B. What are the top 10 physical activity questions in schizophrenia? Disability and Rehabilitation (2016).

20. Keck, P.E., McElroy, S.L. Bipolar disorder, obesity and pharmacotherapy associated weight gain. J. Clin. Psych. 64:12, pp. 1426-35 (2003).

21. Krishnan, K.R. Psychiatric and medical comorbidities of bipolar disorder. Psych. Med. 67:1, pp. 1-8 (2005).

22. Fiagolini, A., Frank, E., Scott, J.A., Turkin, S., Kupfer, D.J. Metabolic syndromes in bipolar disorder: findings from the Bipolar Disorder Centre for Pennsylvanians. Bipolar Disorders. 7:5, pp. 424-30 (2005).

23. Soundy, A., Wampers, M., Probst, M., De Hert, M., Stubbs, B., Vancampfort, D. Physical activity and sedentary behaviour in outpatients with schizophrenia: A systematic review and meta-analysis. Int Jour of Therapy and Rehabilitation. 20:12, pp. 588-96 (2013).

24. Strohle, A. Physical activity, exercise, depression and anxiety disorders. J Neural Transm. 116:777 (2009).

25. Lloyd-Evans, B. In-Patient Alternatives to Traditional Mental Health Acute In-Patient Care. Report for the National Institute for Health Research Service Delivery and Organisation Programme. January 2010.

26. Stubbs, B., Soundy, A., Probst, M., De Hert, M., De Herdt, A., Vancompfort, D. Understanding the role of physiotherapists in schizophrenia: an international perspective from members of IOPTMH. Journal of Mental Health. 23:3, pp. 125-9 (2013).

27. Vancampfort, D., Probst, M., Helvik Skjaerven, L. Systematic review of the benefits of physical therapy within a multidisciplinary care approach for people with schizophrenia. Physical Therapy. 92, pp. 11-23 (2012).

28. Aadahl, M., Jorgensen, T. Validation of a new self-report instrument for measuring physical activity. Med Sci Sports Exerc. 35;7, pp.1196-202 (2003).

29. Bull, F., Maslin, T., Armstrong, T. Global physical activity questionnaire (GPAQ): a nine country reliability and validity study. Journal of Physical Activity and Health. 6, pp.790-804 (2006).

30. Furnham, A. Response bias, social desirability and dissimulation. Personality and Individual Differences. 7:3, 385-400 (1986). 